\title{
Evaluating Outcome Research for Hypersexual Behavior
}

\author{
Joshua B. Grubbs ${ }^{1}$ - Joshua N. Hook ${ }^{2}$ Brandon J. Griffin ${ }^{3}$ - Don E. Davis ${ }^{4}$
}

Published online: 5 July 2015

(C) Springer International Publishing AG 2015

\begin{abstract}
Despite the decision to omit hypersexual behavior or hypersexuality from the Diagnostic and Statistical Manual of Mental Disorders, Fifth Edition (DSM-5), research regarding the nature of hypersexual behavior has flourished in recent years. One area in which there is still a distinct and present need for more work has been outcome research related to the efficacious treatment of hypersexual behavior. Although prior reviews have systematically delineated the necessary changes and improvements in outcome research related to the treatment of hypersexuality, the present review seeks to evaluate the current state of outcome research in light of recent advances in the understanding of hypersexual behavior. Prior reviews are synthesized, recent outcome studies are examined, recent advances in the treatment of related disorders are reviewed, recent advances in hypersexual behavior research are evaluated in terms of treatment implications, and recommendations for future research are offered.
\end{abstract}

Keywords Hypersexual behavior · Sex addiction .

Treatment $\cdot$ Outcome $\cdot$ Pornography

This article is part of the Topical Collection on Sex and Addiction

Joshua B. Grubbs

joshua.grubbs@case.edu

1 Department of Psychological Sciences, Case Western Reserve University, 10900 Euclid Avenue, Cleveland, OH 44106-7123, USA

2 University of North Texas, Denton, TX, USA

3 Virginia Commonwealth University, Richmond, VA, USA

4 Georgia State University, Atlanta, GA, USA

\section{Introduction}

Despite controversies regarding the nature of hypersexual behavior [1] and the omission of a diagnosis from the Diagnostic and Statistical Manual of Mental Disorders, Fifth Edition (DSM-5) [2], the science of hypersexual behavior has proliferated and advanced rapidly in recent years [for reviews, see 3-6]. For example, within the past 5 years alone, investigators have refined the definitions, assessments, and diagnostic precision of hypersexual behavior [7, 8]. Hypersexuality has been linked with personality correlates [9], relationship styles [10], emotional states [11], and even moral concerns [12]. These rapid changes make the half-life of past reviews pertaining to outcome research quite brief, and it is essential that the field maintain an ongoing conversation, so that outcome studies being developed now remain relevant to the likely trajectory of the field. The purpose of this article is to review recent advances in the study of hypersexual behavior in order to discuss implications for outcome research being initiated over the next few years.

Over the past 25 years, scholars proposed numerous treatments for hypersexual behavior, though few have been studied empirically. These treatments include psychotherapeutic interventions [13••], psychopharmacological interventions [14••], as well as a range of case studies delineating mixed treatment methods [15]. Even so, recent reviews highlight methodological problems in many outcome studies $[13 \bullet \bullet, 14 \bullet \cdot]$. For the present review, we (a) synthesize methodological and theoretical reviews of hypersexual behavior treatments published within the past 3 years, (b) explore advances in hypersexual behavior treatments, (c) explore advances in the treatment of related disorders, (d) review recent advances in the psychosocial and neurological understanding of hypersexual behavior with implications for treatment, and (e) provide recommendations for future research related to hypersexual behavior. 


\section{Prior Reviews}

Within the past 3 years, several reviews of hypersexual behavior treatments have been published. These reviews have ranged from methodological critiques $[13 \bullet \bullet, 14 \bullet \cdot]$ to examinations of specific techniques [16] and theoretical integrations $[15,17]$. We summarize the key findings of these prior works, with a focus on the recommendations from previous reviewers.

In a 2013 review, Naficy and colleagues considered the body of research surrounding pharmacological treatments of hypersexual behavior. Although several examples of successful pharmacological treatments for hypersexual behavior were described, the primary conclusions of the review focused on the methodological limitations that hampered pharmacological research. Chiefly, studies were consistently limited by small sample sizes, exclusively male samples (despite numerous studies documenting hypersexual tendencies in women $[18,19]$ ), predominantly Caucasian samples (despite numerous studies documenting hypersexual tendencies in diverse populations $[20,21])$, and discrepancies in inclusion criteria. Naficy and colleagues [14.•] identified only one double-blind, placebo-controlled study that approached conventional standards for rigorous research. Notably, in such a rigorous study, pharmacological interventions performed no better than placebo interventions, casting doubt on the utility of such interventions for the treatment of hypersexual behavior.

In a 2014 review [13••], Hook and colleagues identified 14 studies (8 psychotherapeutic and 6 pharmacological) that examined treatments of hypersexual behavior published within the past 25 years. Although several of these studies were promising, there were a number of methodological problems that hampered the generalizability of findings. Primarily, the majority of studies of the treatment of hypersexual behavior lacked consistent inclusion criteria, with some exclusively relying on self-reported feelings of hypersexual behavior rather than any behavioral measure, while others required strict behavioral criteria to be met. Furthermore, the vast majority of studies lacked diversity in terms of sexual orientation, race, and gender, with most participants being heterosexual, Caucasian males. Most studies also lacked methodological rigor according to conventional standards [22-24], lacked consistency in treatment modalities, and lacked consistency in the reporting of results. In sum, although preliminary research was promising, the body of literature as a whole was sparse and methodologically limited.

Building on Hook and colleague's methodological review [13••], Franqué, Klein, and Briken [16] provided an in-depth review of the techniques used in the treatment of hypersexual behavior, with a focus on the possible etiologies of hypersexual behavior proposed by each respective treatment approach. Although prior studies have mentioned a number of efficacious techniques for treating hypersexual behavior (e.g., acceptance and commitment therapy; cognitive behavioral therapy), Franqué and colleagues [16] concluded that the vast majority of prior research on the topic lacked etiological clarity or focus on the underlying processes that drive hypersexual behavior. In concert with other reviews, they concluded that research related to the treatment of hypersexual behavior is still in its infancy, often lacking methodological rigor and etiological clarity. Collectively, these prior reviews suggest that there is a need for substantially more treatment studies that possess etiological clarity and methodological rigor.

\section{New Outcome Research}

Building on past reviews, we identify recent advances in outcome research related to hypersexual behavior. Specifically, we examine studies of the treatment of hypersexual behavior or related conditions (e.g., compulsive pornography use), with a focus on treatment or outcome studies published within the past 3 years that have not been previously examined by prior reviews on the topic. To this end, a variety of databases were queried including PubMed, ProQuest, JSTOR, PsycInfo, and Medline. Additionally, Google Scholar and Ebsco Host's Academic Search Complete were also queried, as they both sample a range of databases. Search terms included "hypersexuality," "hypersexual behavior," "sex addiction," "sexual addiction," "pornography addiction," "sexual compulsivity," and "sex addict." The search was limited to articles published between January 1, 2012 and March 1, 2015. Additionally, only articles examining the treatment of nonparaphilic hypersexual behavior not better accounted for by medical reasons were reviewed.

Using the previously described methods, only one new study was identified [22-24] that constituted a study of outcome research related to the treatment of hypersexual behavior not previously subsumed by a prior review. In an inpatient treatment setting, Hartwood and colleagues [22-24] described the intensive treatment of individuals with reported sexual addictions in comparison with those who reported sexual addictions and comorbid substance use disorders. In a total treatment sample of 57 (52 men, 5 women), 21 participants reported experiencing only a sexual addiction; whereas, 36 reported experiencing a comorbid sexual addiction (SA) and substance use disorder (SUD). Comorbid substance use included a range of substances (e.g., alcohol, prescription pain medicine, cocaine, heroin). Inpatient treatment for sexual addiction consisted of individual therapy, group therapy, 12-step support, recovery planning, and psychosocial education. Treatment for comorbid SA and SUD followed a similar pattern, with intensive group therapy and 12-step support for SUD as well. After successful completion of the intensive outpatient program $(M=54.75, \mathrm{SD}=13.97)$, participants were discharged 
with a plan in place for continuing care. At 6 months post treatment, both those receiving treatment for SA and for SA with comorbid SUD reported significant continued gains in behavioral regulation, sexual impulse control, and general psychological well-being. Notably, in general, neither treatment group did particularly better or worse than the other on measures of sexual addiction and general well-being. In the SA-SUD treatment group, reductions in substance use were also noted at 6 months post treatment, suggesting that the simultaneous treatment of SA and SUD is a worthwhile endeavor.

This research is particularly salient given that substance use disorder populations are more likely to report problems with hypersexual behavior than the general population [22-25]. Furthermore, prior research has explicitly recommended that substance use disorders demand precedent in treatment over hypersexual behavior [26]. Although the safety concerns presented by a substance use disorder might demand immediate treatment, the results of Hartmann and colleague's [22-24] work suggest that both substance use and hypersexual behavior may be effectively addressed concurrently rather than sequentially. Several of the previously mentioned methodological limitations also applied to Hartmann and colleagues' [22-24] work. Primarily, the sample was predominantly male, and information regarding race and sexual orientation for participants was not provided. Finally, treatment techniques were only briefly described, without a great deal of clarity regarding the specifics of therapeutic approaches. Collectively, these limitations hamper the generalizability of the findings.

\section{Advances in Treatments of Related Disorders}

Moving beyond examinations of the treatment of hypersexual behavior specifically, we noted that recent advances in the treatment of other process addictions (i.e., Internet addiction) may be relevant to the present review. For example, cognitive behavioral therapy and similar approaches are common strategies for treating a wide variety of mental health concerns, including addictions [27]. Within the past 3 years, there have been some advances in the treatment of process addictions using CBT-based techniques that might bear implications for outcome research in the treatment of hypersexual behavior.

A particularly promising line of research related to the treatment of process addictions has been the recent development of CBT for Internet addiction (CBT-IA) [28]. This therapy is designed to help individuals reduce the excessive use of the Internet, including excessive sexual behavior on the Internet. Using a three-phase approach, CBT-IA first seeks to reduce the problematic use with targeted behavioral interventions; it then seeks to modify maladaptive cognitions related to the problematic use, particularly thoughts associated with denial; finally, using a synthesis of $\mathrm{CBT}$ and harm reduction therapy (HRT), CBT-IA seeks to identify and treat any comorbid conditions that are related to the excessive use of the Internet.

Importantly, CBT-IA has shown promise as an effective treatment modality for addressing Internet addiction. In a 12week treatment study of 128 Internet-addicted clients (35\% women, $65 \%$ men), CBT-IA was found to be effective in reducing compulsive use of the Internet and self-reported feelings of addiction [29]. Moreover, the majority of these gains were present both at the end of treatment and 6 months later, demonstrating long-term maintenance of therapeutic gains. Although CBT-IA was not designed to primarily treat sexual disorders, $39 \%$ of the participants in this treatment study reported compulsive use of the Internet for sexual media consumption. The efficacy of CBT-IA in reducing patterns of online compulsive sexual behavior may inform the appropriate treatment of hypersexual behavior more generally.

Similar results have also been found for manualized CBT and motivational interviewing (MI) for Internet addiction [30]. In a small, qualitative analysis of seven individuals seeking treatment for problematic use of the Internet, van Rooij and colleagues found that a manualized CBT approach that integrated MI was effective in reducing feelings of addiction and problematic behaviors over the course of 5-10 sessions. Again, although the design of the study was focused on Internet addiction more broadly, three out of seven participants in the analysis reported compulsive use of online sexual media as their primary difficulty, raising the possibility of the effectiveness of such a treatment pattern for hypersexual concerns as well.

These patterns of results have also been confirmed by a recent meta-analysis on the treatment of Internet addiction [31]. Results from this meta-analysis noted that CBT as a treatment for Internet addiction (including online sexual compulsivity) demonstrated consistent gains, often above and beyond the gains accomplished through other psychotherapy modalities. Again, although the studies in this meta-analysis were not concerned with hypersexual behavior specifically, online sexual compulsivity was included in the analysis, again pointing to the efficacy of tailored CBT-based interventions for the successful treatment of Internet addiction, which may also include certain forms of hypersexual behavior.

\section{Advances in the Understanding of Hypersexuality}

Despite a limited amount of outcome research, there have been a number of recent studies that have enhanced current understandings of hypersexual behavior in ways that may inform treatment. At the forefront of these advances were the diagnostic criteria and etiological considerations that were put forth for consideration in the DSM-5. Although hypersexual behavior was ultimately excluded from the publication, the 
consolidation of diagnostic criteria $[3,32]$, the development of rigorous and comprehensive assessment instruments related to those criteria (e.g., Hypersexual Behavior Inventory, [7]), and a field trial assessing the reliability and validity of the diagnostic criteria [8] provide a consolidated structure that may allow for future advances in the field. Although such advances are still relatively recent, it is likely that they will prove fruitful in guiding future outcome research. Numerous other advances have also been achieved in recent years, with many bearing clear treatment implications.

\section{Advances in Psychosocial Understanding}

Research examining the psychosocial correlates of hypersexual behavior has expanded greatly within the past 3 years. A number of recent works on the correlates of hypersexual behavior have highlighted several common threads [10, 19, 33-36]. Specifically, hypersexual tendencies correlate with a propensity to experience negative emotion [33-39] and poor impulse regulation [19, 40]. Collectively, these findings point toward more global symptoms of dysregulation and distress within hypersexual individuals.

Building on findings linking hypersexual behavior and a propensity toward negative emotion, many works have highlighted deficits in adaptive coping techniques in hypersexual individuals. For example, recent work [41] found that hypersexual tendencies were associated with lower levels of self-forgiveness. Additionally, self-compassion was also lower among those expressing hypersexual tendencies [37]. Mindfulness was also found to be a negative predictor of hypersexual behavior, above and beyond general propensities toward negative emotion [38]. In short, hypersexual individuals seem to have a range of deficiencies in adaptive coping mechanisms, particularly those that involve dealing with negative emotions. These findings are particularly relevant for future treatment research. Mindfulness-based techniques and interventions have demonstrated efficacy in treating addictive disorders such as substance abuse [42] and pathological gambling [43-45], and a wide range of mindfulness-enhancing interventions exist [46]. Similarly, interventions designed to enhance self-compassion [47] are effective in enhancing adaptive coping mechanisms, and interventions based on selfforgiveness [48] have been found to be effective in treating other addictive patterns of behavior and could be tailored for the treatment of hypersexual behavior.

A third advance in the understanding of hypersexual behavior has been the focus of recent works on how many people may identify as struggling with hypersexual behavior (or "sexual addition"), even if their sexual behavior is within normal limits. Indeed, the field has conflated perceived hypersexual behavior with behaviors consistent with problematic hypersexual tendencies, as documented by many theoretical works [1], empirical articles [12, 40], narrative reviews [49] and case studies [15]. A noted shortcoming of research about hypersexual behavior in general is that a number of the inventories designed to measure hypersexual tendencies rely fully on self-reported impressions of one's own behavior, with little attention paid to actual behavior [50]. Even some previously published studies of hypersexual behavior treatment have only relied upon self-reported feelings as inclusion criteria $[13 \bullet \bullet$, $14 \cdot \bullet]$.

Collectively, a body of evidence indicates that there has often been a failure to distinguish between perceived feelings of hypersexuality and actual hypersexual behavior. This shortcoming has hampered the interpretability and general utility of much hypersexual behavior research. Although some have postulated that this weakness is evidence of the faulty nature of hypersexual behavior as a construct [49], we are hesitant to make such a claim. Recent advances in the diagnosis and assessment of hypersexual behavior will likely provide greater structure for future treatment studies. Even so, there is also a body of evidence that suggests that for many people, the perception of hypersexual behavior or sexual addiction is, within itself, problematic [15, 40]. In essence, for some individuals, perceived hypersexual behavior may be the problem, even though their behavior is not inherently problematic. Individuals may have more or less stringent sexual values. For individuals who have very strict values, the threshold for feeling potentially very high levels of distress associated with sexual thoughts or behaviors is likely to be very low [12]. Even if a sexual behavior is infrequent, a person with strict sexual values may ruminate heavily on past sexual behaviors in a way that promotes feelings of distress, perceptions of hypersexuality, and behaviors that perpetuate those feelings. Although the notion of perceived addiction is relatively novel in the realm of hypersexual behavior, it has been studied for several years in substance abuse research $[51,52]$ and process addiction research [53], often predicting future recurrences of problematic behaviors and a wide range of psychological distress indicators.

Given the emergence of perceived hypersexual behavior as a construct and the associations of such a perception with truly problematic mental health symptoms [40], there is a need for research addressing perceived hypersexual behavior, in addition to further research related to the treatment of hypersexual behavior itself. This need is most apparent in two domains. Primarily, there is a need for research that objectively distinguishes between (or at least clearly relates) perceived hypersexual behavior and actual hypersexual tendencies. Secondarily, there is a need for research into the development, etiology, progression, and treatment of perceived hypersexual behavior, particularly in the absence of objective hypersexual tendencies. Indeed, the modification of negative self-perceptions is a hallmark of most modern psychotherapies. For at least some individuals, there is evidence to suggest that the maladaptive self-view associated with perceived hypersexual behavior is 
of greater consequence than their sexual behaviors themselves. As such, there is a need for studies in which perceived hypersexual behavior is effectively controlled for.

\section{Advances in Neurological Understanding of Hypersexuality}

Moving beyond psychosocial associates of hypersexual behavior, recent studies have also suggested that neurological factors may be related to hypersexual behavior $[54,55]$. In particular, functional magnetic resonance imaging (fMRI) has revealed similarities between the way that hypersexual individuals respond to sexual stimuli and the way that substance abusers respond to substances on which they are dependent [54]. Similarly, in a sample of 64 adult males, Kühn and Gallant [55] found that gray matter volume within the reward system structures of the brain (i.e., the right caudate) was inversely correlated with the number of hours an individual reported viewing pornography. Collectively, both of these studies suggest similar neurological structures between hypersexual behavior and other addictive processes. Furthermore, in addition to these studies, recent editorials [56] and theoretical reviews [57] pointed to the importance of understanding neurobiological underpinnings of certain forms of hypersexual behavior. Although some of these opinions have been subject to debate, general consensus exists that implicates neurobiological underpinnings in the experience of hypersexual behavior and associated conditions [58].

Collectively, these recent works point toward an understanding of neurological factors as related to the experience of hypersexual behavior and also highlight the possibility of enhanced pharmacological management of hypersexual behavior. Although prior reviews [14••] have highlighted that poor etiological understanding of hypersexual behavior has limited pharmacological management of the disorder, this emerging body of research is enhancing present understandings of the potential etiology and neurobiology of hypersexual behavior. By extension of this enhanced understanding, it is plausible that more efficacious pharmacological treatments might be tested, and it is likely that this is an area in which productive research might be conducted.

\section{Conclusions and Recommendations}

At the outset of the present review, we sought to examine recent advances in the treatment of hypersexual behavior. In the past, reviews have noted that research on the treatment of hypersexual behavior has been limited both in quantity and methodological quality. Such a trend continues into the present. Despite broad-based searches of research published within the past 3 years, we identified only one new study examining the treatment of non-paraphilic hypersexual behavior [22].
Although this work represents an important contribution, it too suffers from many of the same limitations delineated in prior works. This dearth of new research highlights conclusions of prior reviews calling for more research in this domain as well as more specific needs for greater rigor and control in such research. Even so, there have been recent advances that may inform treatment.

Within the past 3 years, research that may inform the treatment of hypersexual behavior has flourished. As previously reviewed, a number of studies have illuminated various factors relevant to the development and maintenance of hypersexual behavior patterns. Such factors have included (a) advances in the neuropsychological understanding of hypersexual behavior, (b) the considerations of moral emotions and perceptions in hypersexual behavior, and (c) the treatment of theoretically similar disorders. These advances in research around the understanding of hypersexual behavior and related disorders lead to a few very clear recommendations for the field moving forward.

Primarily, there is still a need for more research related to the treatment of hypersexual behavior. The small number of outcome studies illustrates a conclusion made by prior evaluations of the field - the study of hypersexual behavior is still a field in its infancy [5]. Furthermore, the relative absence of empirically based outcome studies has greatly hampered the present understanding of hypersexual behavior. However, as has been highlighted in prior reviews, simple quantity alone is not a sufficient solution to the absence of research on this topic; much more rigorous methodologies must also be employed. For example, the majority of research on both the treatment of hypersexual behavior and the etiology of hypersexual behavior remains distinctly biased toward Caucasian males. Inclusion criteria for outcome studies remain diverse, and the measurement of hypersexual behavior in general still lacks consistency across studies. The calls for greater theoretical and methodological rigor delineated in prior reviews $[13 \bullet \bullet, 14 \bullet \bullet, 16]$ still persist and must be addressed for outcome research to make any substantive advances in the future.

There is also a need for more case studies examining the treatment (both successful and unsuccessful) of hypersexual behavior patterns. Although the psychiatric and medical literature are replete with case studies of the treatment of hypersexual behavior secondary to primary medical diagnosis, there is a relative absence of recent case studies examining either the pharmacological or psychotherapeutic treatment of more general non-paraphilic hypersexual behavior. In the absence of both controlled studies of treatment efficacy and examples of individual treatment techniques and outcomes, there is very little research on which to base any attempt at an evidencebased treatment protocol for hypersexual behavior [59].

Given recent advances in the use of CBT-based techniques for the treatment of Internet addiction, there is a need for research examining the utility of such techniques in reducing 
hypersexual behaviors, particularly those related excessive online sexual behaviors. Given that a substantial portion of individuals who identify as hypersexual engage in excessive pornography consumption online [60], the demonstrated utility of CBT for Internet addiction in reducing problematic online behavior is a promising avenue for addressing certain forms of hypersexual behavior. Future research might focus on determining whether treatments of hypersexual behavior when coupled with other forms of addiction are efficacious as a result of mechanisms that are common to addictive patterns of behavior in general or if unique mechanisms contribute to improvement in process and substance addictions.

Given recent advances regarding perceived hypersexual behavior and personal morality, there is a need for research examining the roles of self-conscious emotions and beliefs in the treatment of hypersexual behavior [39]. As excessive guilt or shame may lead to over-orunder-reporting of hypersexual tendencies, more research needs to effectively control for these variables. There is also a need for research examining how strong moral beliefs around sexual behavior might be effectively addressed in the therapeutic context to promote wellbeing. This may be an area in which the aforementioned interventions addressing self-compassion, mindfulness, and self-forgiveness might be particularly relevant.

Finally, there is a need for outcome research examining specific treatments for specific forms of hypersexual behavior. Prior research has extensively documented that compulsive sexual behavior patterns may vary drastically from person to person. Although the study of hypersexual behavior is a sub-field still in its infancy, diverse expressions of the same disorder might necessitate diverse treatment approaches.

\section{Compliance with Ethics Guidelines}

Conflict of Interest Joshua N. Hook, Brandon J. Griffin, and Don E. Davis declare that they have no conflict of interest.

Joshua B. Grubbs would like to gratefully acknowledge the support of the John Templeton Foundation, Grant \# 36094, in support of the writing of this manuscript.

Human and Animal Rights and Informed Consent This article does not contain any studies with animal subjects performed by any of the authors.

All studies conducted by the authors using human subjects were in accordance with each author's respective institution IRB standards. All procedures performed in studies involving human participants were in accordance with the ethical standards of the institutional and/or national research committee and with the 1964 Helsinki Declaration and its later amendments or comparable ethical standards.

\section{References}

Papers of particular interest, published recently, have been highlighted as:

•• Of major importance

1. Reay B, Attwood N, Gooder C. Inventing sex: the short history of sex addiction. Sex Cult. 2013;17:1-19.

2. Piquet-Pessôa M, Ferreira GM, Melca IA, Fontenelle LF. DSM-5 and the decision not to include sex, shopping or stealing as addictions. Curr Addict Rep. 2014;1:172-6.

3. Kafka MP. The development and evolution of the criteria for a newly proposed diagnosis for DSM-5: hypersexual disorder. Sex Addict Compulsivity. 2013;20:19-26.

4. Kafka MP. What happened to hypersexual disorder? Arch Sex Behav. 2014;43:1259-61.

5. Kor A, Fogel YA, Reid RC, Potenza MN. Should hypersexual disorder be classified as an addiction? Sex Addict Compulsivity. 2013;20:27-47.

6. Reid RC. Personal perspectives on hypersexual disorder. Sex Addict Compulsivity. 2013;20:4-18.

7. Reid RC, Garos S, Carpenter BN. Reliability, validity, and psychometric development of the hypersexual behavior inventory in an outpatient sample of men. Sex Addict Compulsivity. 2011;18:30 51.

8. Reid RC, Carpenter BN, Hook JN, Garos S, Manning JC, Gilliland $\mathrm{R}$, et al. Report of findings in a DSM-5 field trial for hypersexual disorder. J Sex Med. 2012;9:2868-77.

9. Rettenberger M, Klein V, Briken P. The relationship between hypersexual behavior, sexual excitation, sexual inhibition, and personality traits. Arch Sex Behav. 2015. doi:10.1007/s10508-014-0399-7.

10. Gilliland R, Blue Star J, Hansen B, Carpenter B. Relationship attachment styles in a sample of hypersexual patients. J Sex Marital Ther. 2014; Online first edition.

11. Schultz K, Hook JN, Davis DE, Penberthy JK, Reid RC. Nonparaphilic hypersexual behavior and depressive symptoms: a meta-analytic review of the literature. J Sex Marital Ther. 2014;40: 477-87.

12. Grubbs JB, Exline JJ, Pargament KI, Hook JN, Carlisle RD. Transgression as addiction: religiosity and moral disapproval as predictors of perceived addiction to pornography. Arch Sex Behav. 2015;44:125-36.

13.• Hook JN, Reid RC, Penberthy JK, Davis DE, Jennings DJ. Methodological review of treatments for nonparaphilic hypersexual behavior. J Sex Marital Ther. 2014;40:294-308. A detailed methodological review of treatment research of non-paraphilic hypersexual behavior over the past 25 years including both psychotherapeutic and psychopharmacological studies. The authors evaluate the body of research regarding the treatment of hypersexuality and offer detailed suggestions for future improvements.

14.• Naficy H, Samenow CP, Fong TW. A review of pharmacological treatments for hypersexual disorder. Sex Addict Compulsivity. 2013;20:139-53. A detailed review of psychopharmacological treatments for non-paraphilic hypersexual behavior. The authors provide detailed analysis of the shortcomings of prior research and provide recommendations regarding the future of such research.

15. Cantor JM, Klein C, Lykins A, Rullo JE, Thaler L, Walling BR. A treatment-oriented typology of self-identified hypersexual behavior referrals. Arch Sex Behav. 2013;42:883-93.

16. Franqué F, Klein V, Briken P. Which techniques are used in psychotherapeutic interventions for nonparaphilic hypersexual behavior? Sex Med Rev. 2014;3:3-10. 
17. Rosenberg KP, Carnes P, O'Connor S. Evaluation and treatment of sex addiction. J Sex Marital Ther. 2014;40:77-91.

18. Klein V, Rettenberger M, Briken P. Self-reported indicators of hypersexuality and its correlates in a female online sample. J Sex Med. 2014; 11:1974-81.

19. Reid RC, Dhuffar MK, Parhami I, Fong TW. Exploring facets of personality in a patient sample of hypersexual women compared with hypersexual me. J Psychiatr Pract. 2012;18:262-8.

20. Amaral MLS, Abdo CH, Tavares H, Scanavino MDT. Personality among sexually compulsive men who practice intentional unsafe sex in São Paulo, Brazil. J Sex Med. 2014;2: 557-66.

21. Scanavino MDT, Ventuneac A, Rendina HJ, Abdo CH, Tavares H, do Amaral ML, et al. Sexual compulsivity scale, compulsive sexual behavior inventory, and hypersexual disorder screening inventory: translation, adaptation, and validation for use in Brazil. Arch Sex Behav. 2014. doi:10.1007/s10508-014-0356-5.

22. Chambless DL, Hollon SD. Defining empirically supported therapies. J Consult Clin Psychol. 1998;66:7-18.

23. Hartman LI, Ho V, Arbour S, Hambley JM, Lawson P. Sexual addiction and substance addiction: comparing sexual addiction treatment outcomes among clients with and without comorbid substance use disorders. Sex Addict Compulsivity. 2012;19:284-309.

24. Stavro K, Rizkallah E, Dinh-Williams L, Chiasson JP, Potvin S. Hypersexuality among a substance use disorder population. Sex Addict Compulsivity. 2013;20:210-6.

25. Vesga-Lopez O, Schmidt A, Blanco C. Update on sexual addictions. Dir Psychiatry. 2007;27:143-58.

26. Schneider JP, Irons RR. Assessment and treatment of addictive sexual disorders: relevance for chemical dependency relapse. Subst Use Misuse. 2001;36:1795-820.

27. Marlatt GA, Donovan DM, editors. Relapse prevention: maintenance strategies in the treatment of addictive behaviors. 2nd ed. New York: Guilford Press; 2007.

28. Young KS. CBT-IA: the first treatment model for Internet addiction. J Cogn Psychother. 2011;25:304-12.

29. Young KS. Treatment outcomes using CBT-IA with Internetaddicted patients. J Behav Addict. 2013;2:209-15.

30. van Rooij AJ, Zinn MF, Schoenmakers TM, van de Mheen D. Treating Internet addiction with cognitive-behavioral therapy: a thematic analysis of the experiences of therapists. Int J Ment Heal Addict. 2012;10:69-82.

31. Winkler A, Dörsing B, Rief W, Shen Y, Glombiewski JA. Treatment of Internet addiction: a meta-analysis. Clin Psychol Rev. 2013;33:317-29.

32. Carnes PJ, Hopkins TA, Green BA. Clinical relevance of the proposed sexual addiction diagnostic criteria: relation to the sexual addiction screening test-revised. J Addict Med. 2014;8:450-61.

33. Carpenter BN, Reid RC, Garos S, Najavits LM. Personality disorder comorbidity in treatment-seeking men with hypersexual disorder. Sex Addict Compulsivity. 2013;20:79-90.

34. Egan V, Parmar R. Dirty habits? Online pornography use, personality, obsessionality, and compulsivity. J Sex Marital Ther. 2013;39: 394-409.

35. Reid RC, Cooper EB, Prause N, Li DS, Fong TW. Facets of perfectionism in a sample of hypersexual patients. J Nerv Ment Dis. 2012;200:990-5.

36. Pinto J, Carvalho J, Nobre PJ. The relationship between the FFM personality traits, state psychopathology, and sexual compulsivity in a sample of male college students. J Sex Med. 2013;10:1773-82.

37. Reid RC, Temko J, Moghaddam JF, Fong TW. Shame, rumination, and self-compassion in men assessed for hypersexual disorder. $\mathrm{J}$ Psychiatr Pract. 2014;20:260-8.

38. Reid RC, Bramen JE, Anderson A, Cohen MS. Mindfulness, emotional dysregulation, impulsivity, and stress proneness among hypersexual patients. J Clin Psychol. 2014;70:313-21.
39. Gilliland R, South M, Carpenter BN, Hardy SA. The roles of shame and guilt in hypersexual behavior. Sex Addict Compulsivity. 2011;18:12-29.

40. Grubbs JB, Volk F, Exline JJ, Pargament KI. Internet pornography use: perceived addiction, psychological distress, and the validation of a brief measure. J Sex Marital Ther. 2015;41:83-106.

41. Hook JN, Farrell JE, Davis DE, Van Tongeren DR, Griffin BJ, Grubbs J, et al. Self-forgiveness and hypersexual behavior. Sex Addict Compulsivity. 2015;22:59-70.

42. Bowen S, Witkiewitz K, Clifasefi SL, Grow J, Chawla N, Hsu SH, et al. Relative efficacy of mindfulness-based relapse prevention, standard relapse prevention, and treatment as usual for substance use disorders: a randomized clinical trial. JAMA Psychiatry. 2014;71:547-56.

43. de Lisle SM, Dowling NA, Allen JS. Mindfulness-based cognitive therapy for problem gambling. Clin Case Stud. 2011;10:210-28.

44. de Lisle SM, Dowling NA, Allen JS. Mindfulness and problem gambling: a review of the literature. J Gambl Stud. 2012;28:719-39.

45. Toneatto T, Vettese L, Nguyen L. The role of mindfulness in the cognitive-behavioural treatment of problem gambling. J Gambl Issues. 2007;19:91-100.

46. Segal ZV, Williams JMG, Teasdale JD. Mindfulness-based cognitive therapy for depression. New York: Guilford Press; 2012.

47. Neff KD, Germer CK. A pilot study and randomized controlled trial of the mindful self-compassion program. J Clin Psychol. 2013;69: $28-44$.

48. Scherer M, Worthington Jr EL, Hook JN, Campana KL. Forgiveness and the bottle: promoting self-forgiveness in individuals who abuse alcohol. J Addict Dis. 2011;30:382-95.

49. Ley D, Prause N, Finn P. The emperor has no clothes: a review of the 'pornography addiction' model. Curr Sex Health Rep. 2014;6: 94-105.

50. Womack SD, Hook JN, Ramos M, Davis DE, Penberthy JK. Measuring hypersexual behavior. Sex Addict Compulsivity. 2013;20:65-78.

51. Eiser JR, Van der Pligt J, Raw M, Sutton SR. Trying to stop smoking: effects of perceived addiction, attributions for failure, and expectancy of success. J Behav Med. 1985;8:321-41.

52. Okoli CT, Richardson CG, Ratner PA, Johnson JL. Non-smoking youths" "perceived" addiction to tobacco is associated with their susceptibility to future smoking. Addict Behav. 2009;34:1010-6.

53. Allegre B, Souville M, Therme P, Griffiths M. Definitions and measures of exercise dependence. Addict Res Theory. 2006;14: 631-46.

54. Voon V, Mole TB, Banca P, Porter L, Morris L, Mitchell S, et al. Neural correlates of sexual cue reactivity in individuals with and without compulsive sexual behaviours. PLoS One. 2014;9: e102419.

55. Kühn S, Gallinat J. Brain structure and functional connectivity associated with pornography consumption: the brain on porn. JAMA Psychiatry. 2014;71:827-34.

56. Hilton Jr DL, Watts C. Pornography addiction: a neuroscience perspective. Surg Neurol Int. 2011;2:19.

57. Hilton Jr DL. Pornography addiction - a supranormal stimulus considered in the context of neuroplasticity. Socioaffective Neurosci Psychol. 2013. doi:10.3402/snp.v3i0.20767.

58. Reid RC, Carpenter BN, Fong TW. Neuroscience research fails to support claims that excessive pornography consumption causes brain damage. Surg Neurol Int. 2011;2:64.

59. Kazdin AE. Evidence-based treatment and practice: new opportunities to bridge clinical research and practice, enhance the knowledge base, and improve patient care. Am Psychol. 2008;63:146-59.

60. Sutton KS, Stratton N, Pytyck J, Kolla NJ, Cantor M. Patient characteristics by type of hypersexual behavior referral: a quantitative chart review of 115 consecutive male cases. J Sex Marital Ther. 2014; Online First Edition. 